\title{
Imaging Subtle Microstructural Variations in Ceramics With Precision Ultrasonic Velocity and Attenuation Measurements
}

Edward R. Generazio, Don J. Roth, and George Y. Baaklini Lewis Research Center Cleveland, Ohio 


\section{Summary}

Acoustic images of silicon carbide ceramic disk were obtained using a precision scanning contact pulse echo technique. Phase and cross-correlation velocity, and attenuation maps were used to form color images of microstructural variations. These acoustic images reveal microstructural variations not observable with $\mathrm{x}$-ray radiography.

\section{Introduction}

There is an international research effort to incorporate ceramic components into hot sections of heat engines. A major portion of this effort is directed towards the understanding and control of ceramic processing so that the strength of ceramics may be optimized. To date, the strength of sintered ceramics (e.g., SiC) is well below, by about two orders of magnitude, the theoretical strength (refs. 1 and 4). This discrepancy is understood to be due to the presence of voids, inclusions, agglomerates, and anomalously large grains (ref. 4). These defects, causing premature failure, are introduced or formed during the ceramic manufacturing process. Considerable work has already been done to remove these strength reducing material variations. This has resulted in a steady increase in the fracture strength of ceramics, however, the rate of this increase has slowed. Adding to the problem is the fact that the fracture strength of identically produced experimental samples varies as much as 35 percent (ref. 2). As a result of the loss of momentum toward higher strengths, researchers are turning to ceramic-ceramic fiber composites. These composites show promise of increasing the fracture strength of ceramic materials even further. It is likely that the same material strength variations will be present, at least locally in the matrix, in ceramic composites. Therefore, before abandoning monolithic ceramics for ceramic composites, it would seem appropriate to investigate the origin of these strength reducing defects causing the disparity between the experimental and theoretical fracture strengths for monolithic ceramics. The wide spread in the fracture strengths of identically produced samples is quite troublesome and is of particular interest.

The work shown here is aimed at precision measurement of material variations. The variations that we will be most concerned with are subtle porosity and grain size distribution variations that occur during processing. These variations are essentially unobservable using standard evaluation techniques such as optical or $\mathrm{X}$-ray analysis. These subtle variations may collectively lead to the creation of critical sized pores, grains, or agglomerates.

In order to measure material variations with precision we need a probe that will interact with these variations. Optical and $x$-ray analysis are believed to yield sufficiently accurate results. This is well supported with past and current research results in metals. It should be pointed out that metals also have fracture strengths well below their theoretical strengths. This has been attributed to the presence of dislocations in the metals. Ceramics are extremely sensitive to even slight variations in the microstructure. Direct observation and determination of localized failure sites (usually identified to be isolated pores, inclusions, agglomerates, and anomalously large grains) have shown the importance of these material variations (ref. 4). These failure sites cannot be determined a priori by optical and $\mathrm{x}$-ray methods. It seems plausible that these failure causing variations do not occur spontaneously in isolation but are the result of processing variations or history of processing variations of the environment that surrounds them.

Ultrasonic waves interact substantially with pores, grains, agglomerates, and inclusions. For this reason, ultrasound is chosen as a probe for microstructural variations. Ultrasonic imaging has been used successfully for many years. Imaging techniques such as $\mathrm{C}$-scans for materials and medical sonoscans have proved to be useful. These imaging techniques are imprecise and not sufficiently sensitive to reveal subtle changes in microstructure.

In this work we will demonstrate that by using precise and accurate ultrasonic measurements, microstructural variations unobservable by $\mathrm{x}$-ray techniques can be revealed. These microstructural variations are likely to be present in all sintered ceramic materials.

\section{Interaction of Ultrasonic Waves with Material Variations}

Ultrasonic waves are known to interact with both pores and grains existing in materials. Physically each pore or grain acts as an acoustic scattering site that removes energy from the impeding ultrasonic wave. Additionally, the velocity of the ultrasonic wave is found to be linearly dependent on the bulk density (refs. 5 and 6), while independent of the grain size in a polycrystalline solid. 
The use of pulse-echo techniques is well established. The configuration is shown in figure 1(a). A single broadband ultrasonic pulse (main pulse) is excited (via the crystal) in the buffer rod. The ultrasonic pulse is partially reflected at the buffer rod-couplant-sample (BCS) interface. The first frontsurface reflected pulse is labeled $\mathrm{FS}_{2}$ in figure 1(a). The main pulse travels forward through the sample and then reflects off the back surface of the sample and again interacts with the BCS interface. This first back-surface reflection is labeled $B_{1}{ }^{\prime}$. Echo $B_{1}{ }^{\prime}$ is partially reflected at the BCS interface and is subsequently reflected again at the back surface. The second back-surface reflection is labeled $\mathrm{B}_{2}{ }^{\prime}$. Echo's $\mathrm{B}_{1}{ }^{\prime}$ and $\mathrm{B}_{2}{ }^{\prime}$ are not directly measurable with the $\mathrm{BCS}$ configuration. Their reduced waveform amplitudes, labeled $B_{1}$ and $B_{2}$ in figure 1(b), are measurable.

\section{Attenuation}

The attenuation coefficient for this experimental configuration is given by

$$
\alpha(f)=\frac{1}{2 \Delta X} \ln \left\{\frac{\left|B_{1}(f)\right||R(f)|}{\left|B_{2}(f)\right|}\right\}
$$

with

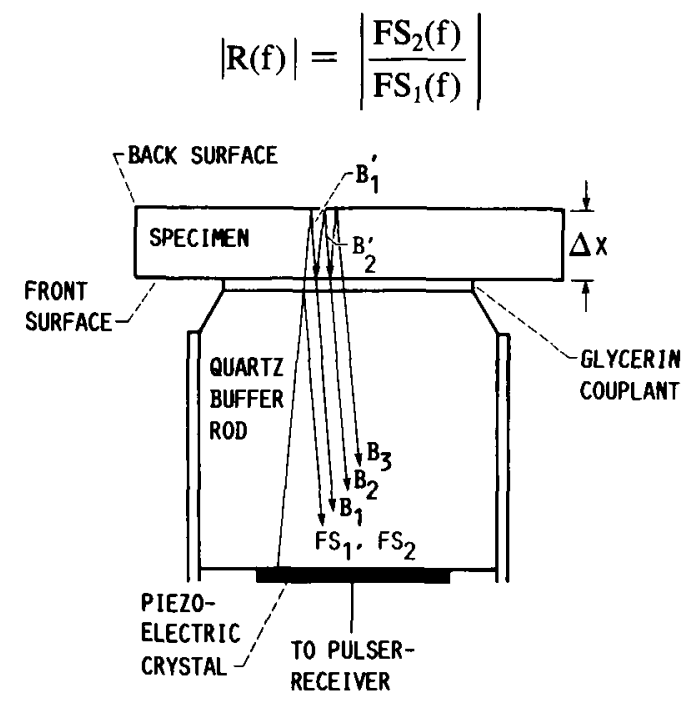

(A)

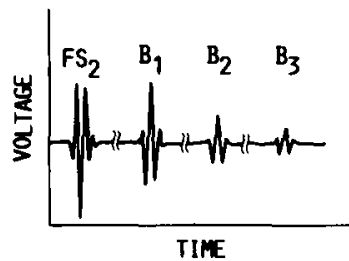

Figure 1.-Diagram of transducer/specimen configuration for pulse echo ultrasonics.

(CD-87-26970)
$\left|\mathrm{FS}_{1}(\mathrm{f})\right|$ and $\left|\mathrm{FS}_{2}(\mathrm{f})\right|$ are the Fourier spectra of the first frontsurface reflection without and with the sample present on the buffer rod, respectively. $|R(f)|$ is the frequency dependent BCS reflection coefficient (ref. 7). $\left|B_{1}(f)\right|$ and $\left|B_{2}(f)\right|$ are the Fourier spectra of pulses $B_{1}$ and $B_{2}$, respectively. $\Delta X$ is the thickness of the sample.

\section{Ultrasonic Velocity}

There are several ways to measure the velocity with the BCS configuration. Peak detection, echo overlap, cross correlation, and phase methods are available (refs. 8 and 9). Both peak detection and echo overlap are the most common used, however, they are rather imprecise. These two techniques are accurate if the ultrasonic pulse shape is not distorted as it traverses the sample. They should not be expected to yield accurate results for most acoustically dispersive materials where the pulse shape is often distorted as it travels through the material. Cross correlation is probably somewhat better than the previous two in that it may be used with noisy signals such as those found with composites. It is, nevertheless "essentially" a mathematical formulation of echo overlap. Phase velocity is the velocity at a particular frequency and is most often expressed as velocity as a function of frequency. The determination phase velocity is independent of the pulse shape and will yield accurate results for both nondispersive and dispersive materials.

Since phase and cross-correlation velocities are probably the most accurate of the four we will use these for the formation of images constructed by mapping velocity over the specimen.

The cross-correlation velocity is given by

$$
\mathrm{V}=\frac{2 \Delta \mathrm{X}}{\tau_{\mathrm{o}}}
$$

where $\tau_{\mathrm{o}}$ is the time shift for which

$$
\left|\lim _{\mathrm{T} \rightarrow \infty} \int_{-\mathrm{T}}^{\mathrm{T}} \mathrm{B}_{1}(\mathrm{t}) \cdot \mathrm{B}_{2}(\mathrm{t}+\tau) \mathrm{dt}\right|-\infty \leq \tau \leq \infty
$$

reaches a maximum value.

The phase velocity is given by

$$
\mathrm{V}(\mathrm{f})=\frac{(2 \Delta \mathrm{X}) 2 \pi \mathrm{f}}{|\Delta \theta|}, \quad \Delta \theta=\theta_{2}-\theta_{1}
$$

with

$$
\theta_{1}(f)=\tan ^{-1}\left\{\frac{\operatorname{Mm}\left(\mathrm{B}_{1}(\mathrm{f})\right)}{\operatorname{Re}\left(\mathrm{B}_{1}(\mathrm{f})\right)}\right\}
$$


and

$$
\theta_{2}(\mathrm{f})=\tan ^{-1}\left\{\frac{\operatorname{lm}\left(\mathrm{B}_{2}(\mathrm{f})\right)}{\operatorname{Re}\left(\mathrm{B}_{2}(\mathrm{f})\right)}\right\}
$$

\section{Experimental}

\section{Samples}

Two disks $38 \mathrm{~mm}$ diameter by $4 \mathrm{~mm}$ were produced using standard ceramic processing techniques. The samples were sintered $0.5 \mathrm{hr}$ at 2090 and $2190^{\circ} \mathrm{C}$ yielding densities of 2.97 and $3.12 \mathrm{gm} / \mathrm{cm}^{3}$, respectively. (The theoretical density for these samples is $3.2 \mathrm{gm} / \mathrm{cm}^{3}$.) After sintering the disks were machined to $\pm 0.0011 \mathrm{~cm}$. One side of each disk was polished to a mirror finish with $3 \mu \mathrm{m}$ diameter diamond slurry. The polishing was done to reduce the acoustic reflection coefficient that subsequently increased the accuracy of the ultrasonic measurement (ref. 7).

\section{Radiography}

Radiographs of the samples investigated were made using both conventional and microfocus techniques. Two methods were used in an effort to obtain the best, highest contrast, radiographs. The results of the two methods were similar. The microfocus radiograph is magnified (as obtained) for contact print processing and for this reason was chosen for comparison with ultrasonic data. The radiographs were made with a molybdenum target with 17 to $21 \mathrm{keV}$ photon energies.

\section{Acoustic Scanning Setup}

The disks were mounted in a polymethalmethacrylate sample holder as shown in figure 2 . A large $\mathrm{O}$-ring was glued to the underside of the sample holder. The sample holder was mounted on a computer controlled $\mathrm{X}-\mathrm{Y}-\mathrm{Z}$ position microscanning table having a step resolution of $1.0 \mu \mathrm{m}$ in the $X, Y$, and $Z$ directions. All scans were done in a 46 by 46 point array with each point separated by $1000 \mu \mathrm{m}$. Above the microscanning table, a $100 \mathrm{MHz}$ broadband transducer having a 1/4 in. diameter buffer rod and $1 / 8$ in. diameter piezoelectric crystal was mounted on a displacement pressure gauge. The contact pressure was $10 \pm 0.1 \mathrm{lb}$.

\section{Data Acquisition, Instrument Control, and Analysis}

It is common to have a computer control movement of instruments, acquire data, and analyze the data. This often excessively burdens the computer and subsequently the researcher forfeits resolution and/or accuracy of the data in order to reduce the acquisition and analysis time and the data storage space required. A problem that pushes the researcher in this direction, is that computer control of instruments and data acquisition is easily done in Basic programming language with standard general purpose interface buss (GPIB) instrument

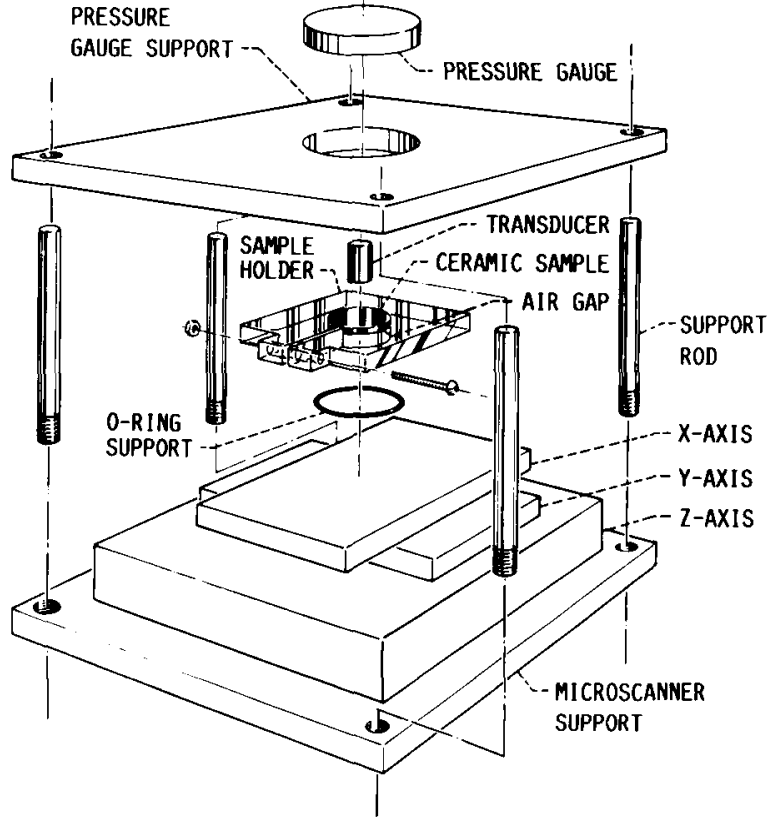

Figure 2.-Diagram of experimental setup used for scanning ceramic samples.

(CD-87-26663)

interface software. This results in a Basic data file that is ready to be analyzed using Basic language mathematics. Fortran programming language would be a more efficient language for this analysis. Computers that are dedicated to instrument control and data acquisition are usually single user systems with one operating system and one programming language. So that, conversion of Basic data files to Fortran data files for high-speed analysis is of little use unless an alternate computer system with Fortran is available.

To optimize the data acquisition and analysis two computers were interfaced for this work. A block diagram of the interface arrangement is shown in figure 3 . Computer $B$ is programmed using Basic language to perform all the instrument control and data acquisition. Computer A contains an array processor (for fast Fourier transforms) and is programmed using Fortran language to perform all the analysis. The acquired data (512

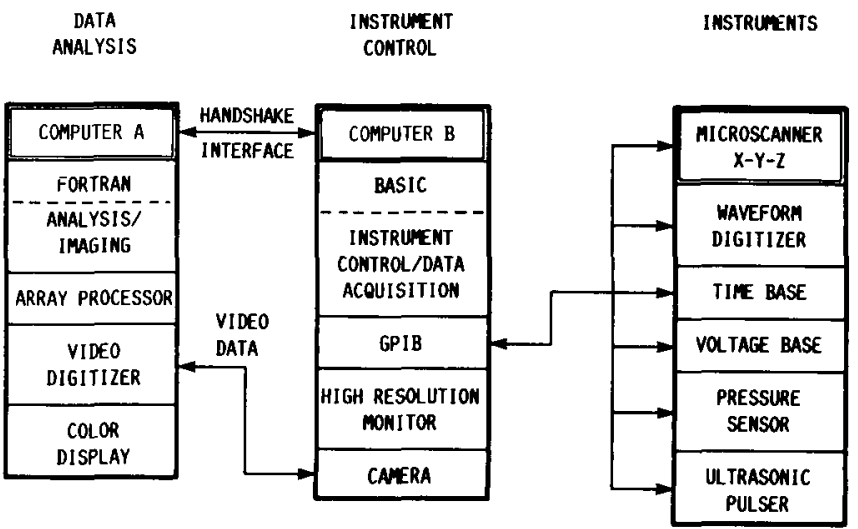

Figure 3.-Diagram of computer configuration used for signal acquisition and analysis. 


\section{ORIGINAL PAGE \\ COLOR PHOTOGRAPH}

point waveform) in computer B is then sent to computer A. A handshake interface between the two computers controls the flow and checks the integrity of the data. The conversion of Basic data to Fortran data is done by an analog video interface.

\section{Results}

The first set of data (figs. 4 to 10) are for the sample sintered at $2090{ }^{\circ} \mathrm{C}$. The radiograph (fig. 4) indicates that the sample is relatively uniform in density. Slight variations can be identified by the trained eye just to the lower left of the center of the sample.

The cross-correlation velocity image (fig. 5) reveals different results from the radiograph. There exists a low-speed area (cloud) near the center of the sample. A velocity scan horizontally across the center of the sample is shown in the figure. The velocity increases fairly uniformly from the center of this cloud out of the edge of the sample. The highest velocities are at the outer edge. The cross-correlation velocity varies from 1.12 to $1.15 \mathrm{~cm} / \mu$ s or about 2.6 percent over the entire sample.

The phase velocity at $100 \mathrm{MHz}$ (fig. 6) reveals a more detailed picture of the velocity changes over the sample. This image indicates that the velocity is varying nonuniformly over the sample. A velocity scan horizontally across the center of the sample is shown in the figure. The velocity oscillates between higher and lower speeds in the center of the sample with the highest velocities at the outer edge. The phase velocity varies from 1.07 to $1.15 \mathrm{~cm} / \mu$ s or about 8 percent over the entire sample.

The attenuation at $80 \mathrm{MHz}$ is shown in figure 7 . The crescent area on the right hand side represents a region having higher attenuation than the central region. The extreme left reveals a curved shaped attenuation band where the attenuation is similar to the central region. The attenuation at $80 \mathrm{MHz}$ varies from 0.0 to $2.0 \mathrm{~Np} / \mathrm{cm}$ over the sample. Zero attenuation

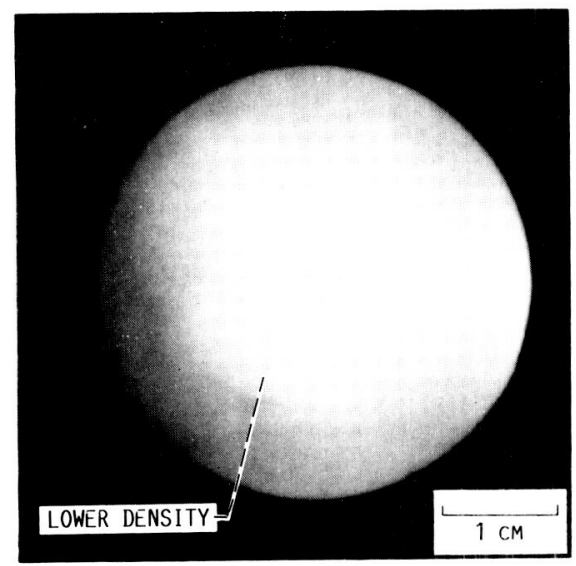

Figure 4.-Microfocus x-ray radiograph of $\mathrm{SiC}$ disk sintered at $2090{ }^{\circ} \mathrm{C}$. (CD-87-26973)

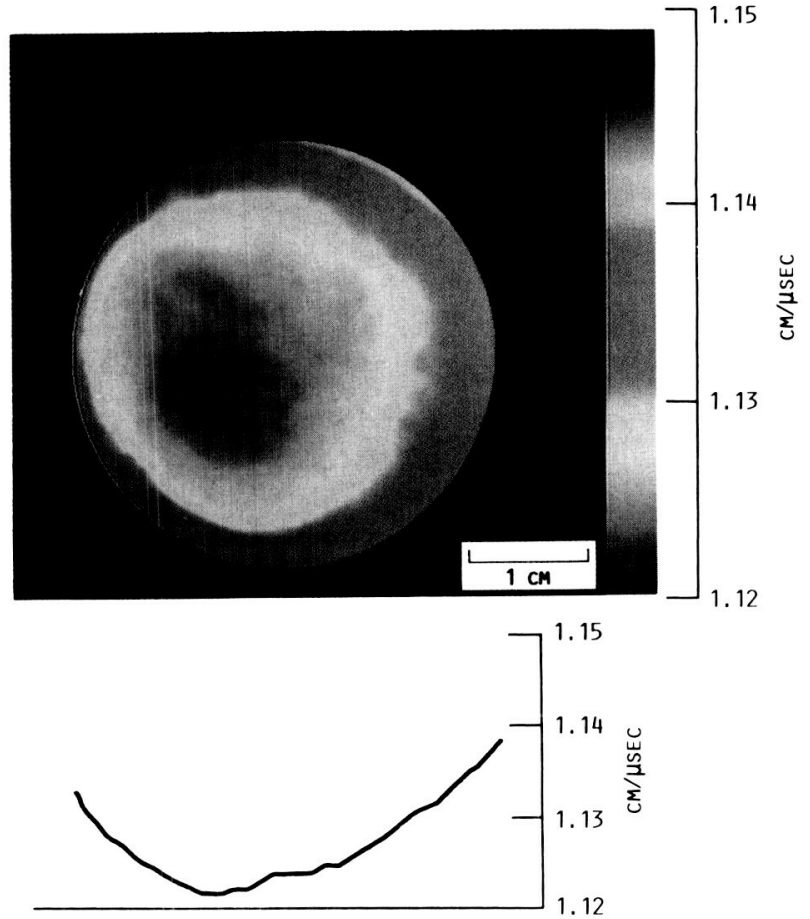

Figure 5. - Cross correlation velocity map of $\mathrm{SiC}$ disk sintered at $2090{ }^{\circ} \mathrm{C}$.

(CD-87-26969)

corresponds to an unmeasurable low attenuation for this frequency and experimental configuration.

At $100 \mathrm{MHz}$ (fig. 8) the relatively uniform crescent region on the right hand side of the image obtained at $80 \mathrm{MHz}$ (fig. 7)
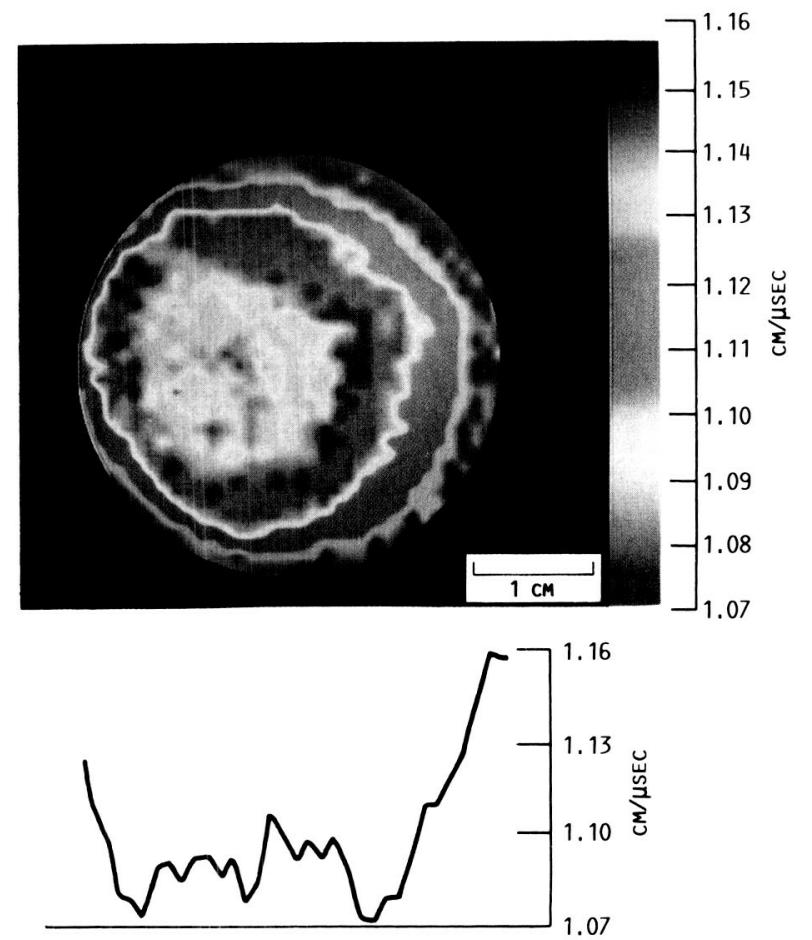

Figure 6.-Phase velocity map of $\mathrm{SiC}$ disk sintered at $2090{ }^{\circ} \mathrm{C}$.

(CD-87-26960) 


\section{ORIGINAL PAGE COLOR PHOTOGRAPH}

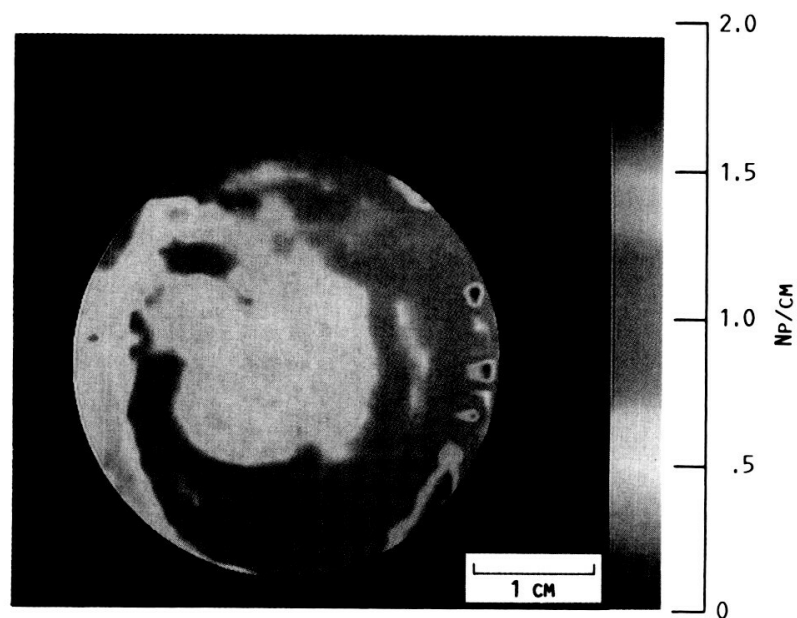

Figure 7.-Attenuation map at $80 \mathrm{MHz}$ for SiC disk sintered at $2090{ }^{\circ} \mathrm{C}$ (CD-87-26966)
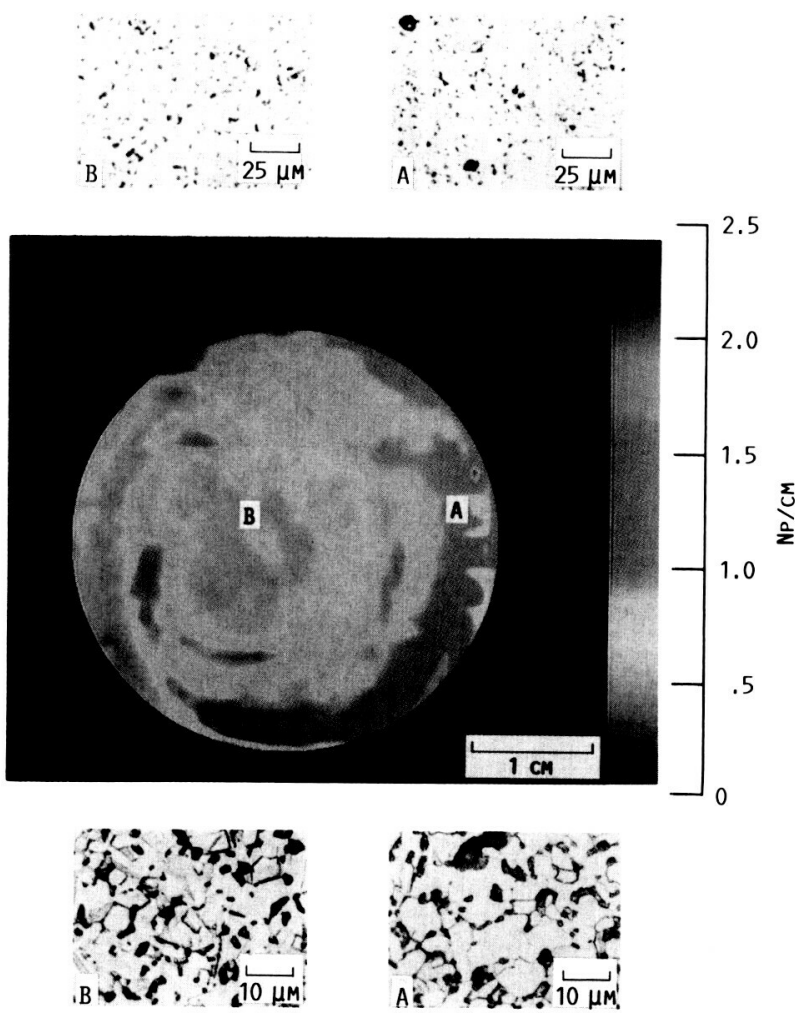

Figure 8.-Attenuation map at $100 \mathrm{MHz}$ and microstructure for SiC disk sintered at $2090^{\circ} \mathrm{C}$.

(CD-87-26964)

now exhibits oscillations in the attenuation that give a spiral galaxy appearance. Note also that the scale is different from the image obtained at $80 \mathrm{MHz}$. The microstructure at point $A$ and $B$ is also shown in figure 8. The ultrasonic direction is vertical. An estimate of the porosity fraction can be made, from the photomicrographs (upper two) of the unetched areas, by determining the ratio of dark areas (pores) to the total area containing these pores. For points $\mathrm{A}$ and $\mathrm{B}$ these ratios are
0.12 and 0.17 , respectively. This indicates that the central region is more porous than the outer right hand side edge. The pore size at point B is about 1 to $5 \mu \mathrm{m}$. Pores about five times larger $(15 \mu \mathrm{m})$ appear scattered throughout the region at point $\mathrm{A}$. The grain size (lower two photomicrographs) at both points $\mathrm{A}$ and $\mathrm{B}$ is approximately the same at 5 to $10 \mu \mathrm{m}$.

The attenuation at $120 \mathrm{MHz}$ (fig. 9) shows a larger central region. The highest attenuation appears as a crescent area on the right hand side edge. The lowest attenuation areas are identified as a crescent area on the left hand side and a central region having a radius of one-half the sample diameter.

As indicated previously, the reflection coefficient can be optimized to obtain the most accurate results. The reflection coefficient is shown in figure 10 . The reflection coefficient over the entire sample ranges from 0.37 to 0.63 . This reflection coefficient, obtained by mirror polishing the sample, yields the most accurate data. Reflection coefficients above 0.8 or below 0.2 result in increased uncertainties in the observed attenuation (ref. 7).

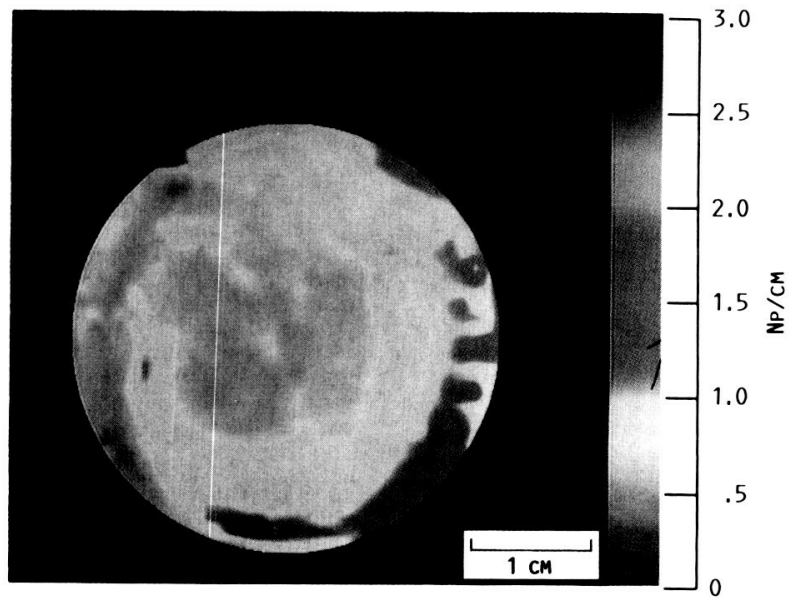

Figure 9.-Attenuation map at $120 \mathrm{MHz}$ for $\mathrm{SiC}$ disk sintered at $2090{ }^{\circ} \mathrm{C}$. (CD-87-26968)

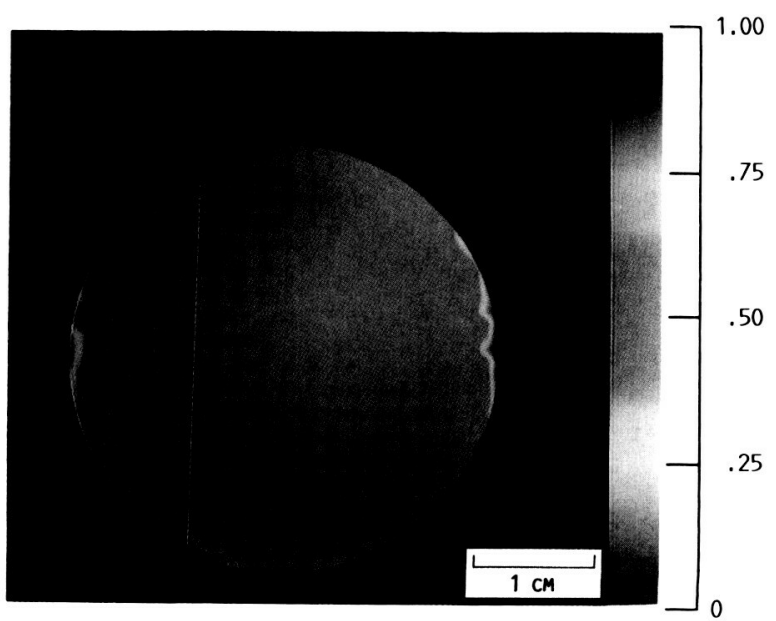

Figure 10.-Reflection coefficient map at $100 \mathrm{MHz}$ for $\mathrm{SiC}$ disk sintered at $2090{ }^{\circ} \mathrm{C}$

(CD-87-26967) 


\section{ORIGINAL PAGE COLOR PHOTOGRAPH}

The next set of data (figs. 11 to 16) are for the sample sintered at $2190{ }^{\circ} \mathrm{C}$. The radiograph (fig. 11) reveals a lowdensity region at the center and extends to the top, left, and lower left. There is a high-density crescent-shaped area, on the right side, that extends to form a ring around the outer edge of the sample. Some higher density strands are present in the central low-density region.

The cross-correlation velocity (fig. 12) reveals a low-speed region in the same position as the low-density region of the $x$-ray data. Two features can be observed, within this lowspeed area, at the same location as the high-density strands in the $\mathrm{x}$-ray data. The velocity horizontally across the center of the sample is also shown in the figure. The velocity is

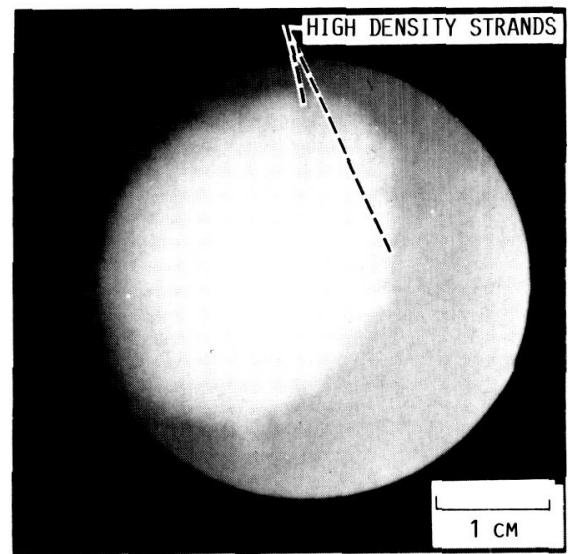

Figure 11.-Microfocus x-ray radiograph of SiC disk sintered at $2190{ }^{\circ} \mathrm{C}$. (CD-87-26972)
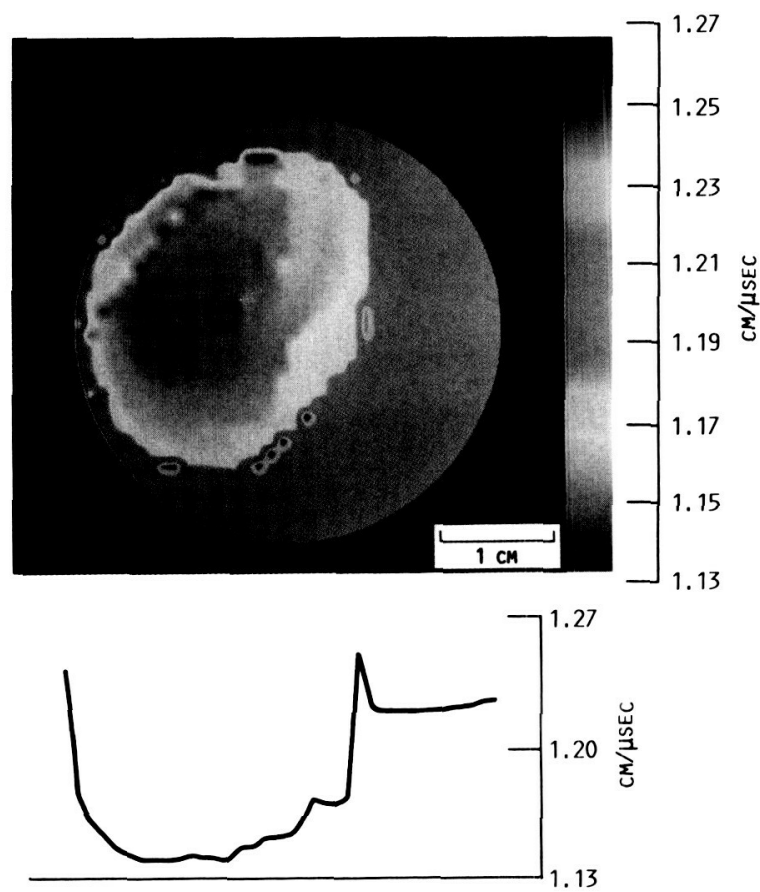

Figure 12.-Cross correlation velocity map of $\mathrm{SiC}$ disk sintered at $2190{ }^{\circ} \mathrm{C}$. (CD-87-26961) relatively uniform in the low-speed region and increases rapidly near the edge of this low-speed area. The cross correlation varies from 1.13 to $1.27 \mathrm{~cm} / \mu \mathrm{s}$ or about 12 percent.

The $100 \mathrm{MHz}$ phase velocity (fig. 13) again reveals a more detailed picture. The phase velocity horizontally across the center of the sample reveals a complicated oscillatory structure with a low-speed center surrounded by a higher speed donutshaped ring. The outer edge of the low-speed region is surrounded by isolated and connected very high-speed areas. The phase velocity varies from 1.12 to $1.24 \mathrm{~cm} / \mu$ s or about 10 percent.

The attenuation at $100 \mathrm{MHz}$ (fig. 14) shows a high attenuation region at the same position as the low-density region of the $\mathrm{x}$-ray data. This high attenuation area has a complicated structure where the attenuation varies rapidly with position from 0.0 to $4.0 \mathrm{~Np} / \mathrm{cm}$. The attenuation in the crescent-shaped area on the right hand side is unmeasurably low.

The microstructure at points $\mathrm{A}$ and $\mathrm{B}$ is shown in figure 14 . The porosity fraction for points $A$ and $B$ is 0.02 and 0.17 , respectively. As in the previous sample the central region is more porous. Note that for point B (top photomicrograph) the left hand side of the photomicrograph exhibits a lower porosity than the right hand side of the photomicrograph. The pore sizes are in the range 2 to $10 \mu \mathrm{m}$. This extreme local variation in porosity was observed horizontally across the high attenuating region. At point $\mathrm{A}$ the microstructure is nearly fully dense with sparse large pores being about $3 \mu \mathrm{m}$. The grain size at points A and B were similar at about 5 to $15 \mu \mathrm{m}$. Several anomalously large grains (fig. 15) were found scattered across
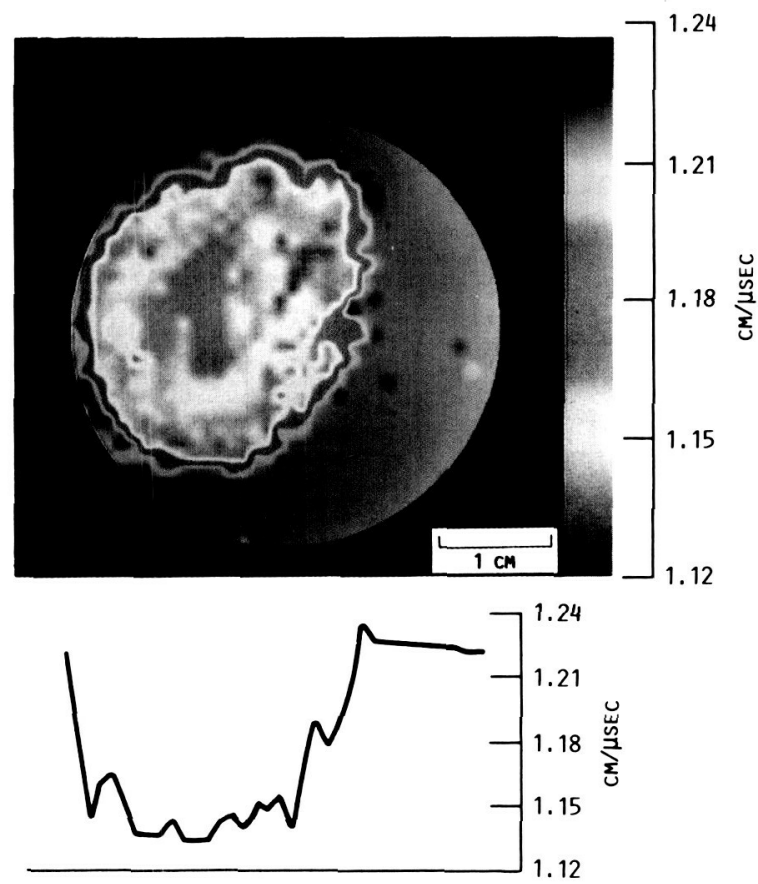

Figure 13.-Phase velocity map of $\mathrm{SiC}$ disk sintered at $2190{ }^{\circ} \mathrm{C}$.

(CD-87-26962) 

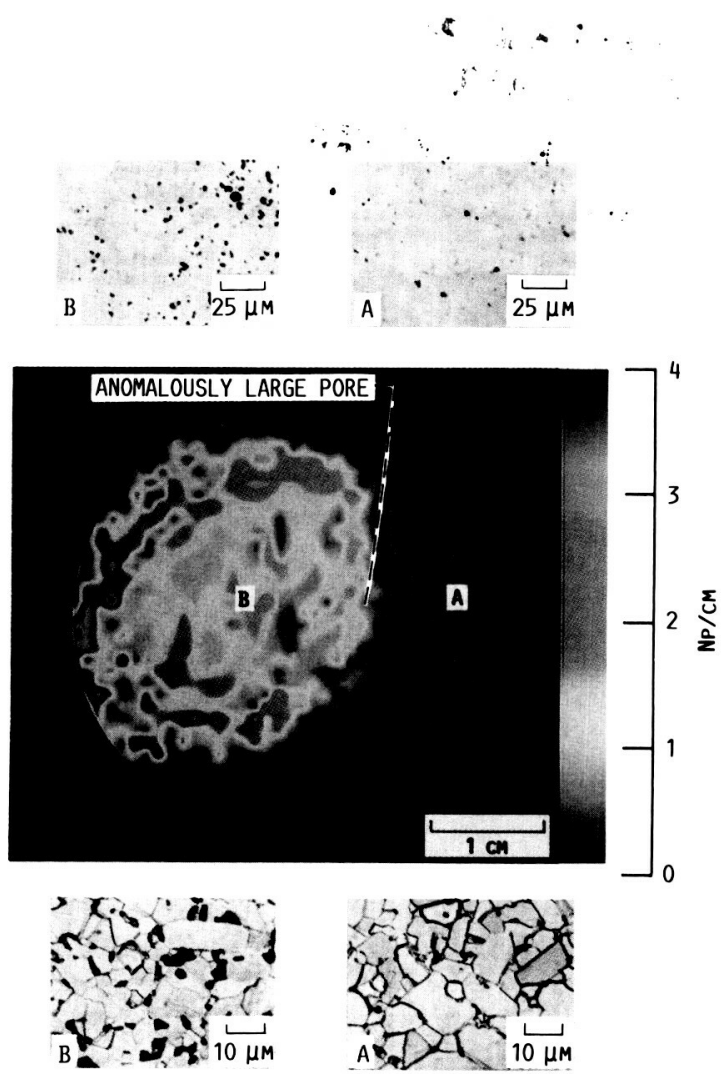

Figure 14.-Attenuation map at $100 \mathrm{MHz}$ and microstructure for SiC disk sintered at $2190{ }^{\circ} \mathrm{C}$.

(CD-87-26963)
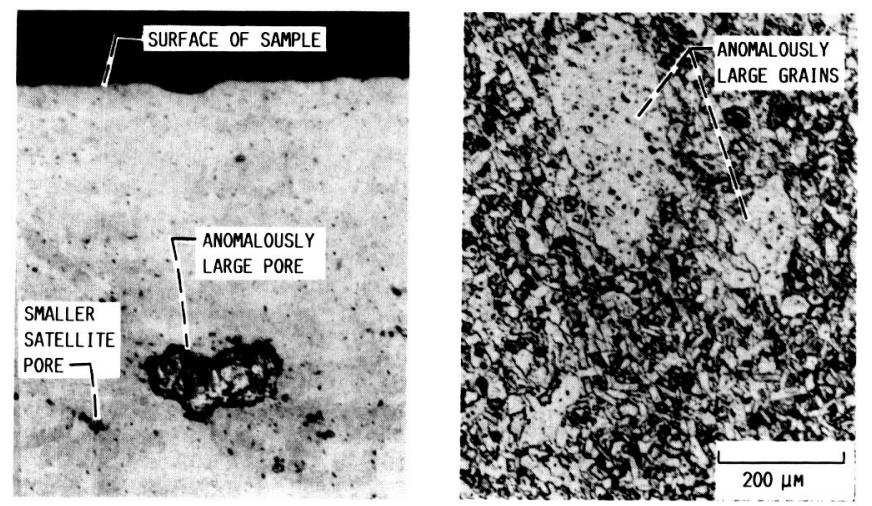

Figure 15.-Anomalously large pore and grains observed in the SiC disk sintered at $2190{ }^{\circ} \mathrm{C}$.

(CD-87-26974)

the line passing through points A and B. In this same path, an anomalously large pore surrounded by smaller satellite pores, was found (fig. 15) at the right hand edge of the high attenuation region.

The sample generally reveals a reflection coefficient (fig. 16) in the range 0.3 to 0.55 . The two large horizontal bands near the center correspond to reflection coefficients in the range 0.7 to 0.85 . They are, for the most part, within range to yield accurate results. There are also, dilute, isolated regions, positioned at the outer edge of the high attenuating region, exhibiting a low reflection coefficient. These areas will yield attenuation results that are of questionable validity.

\section{ORIGINAL PAGE COLOR PHOTOGRAPH}

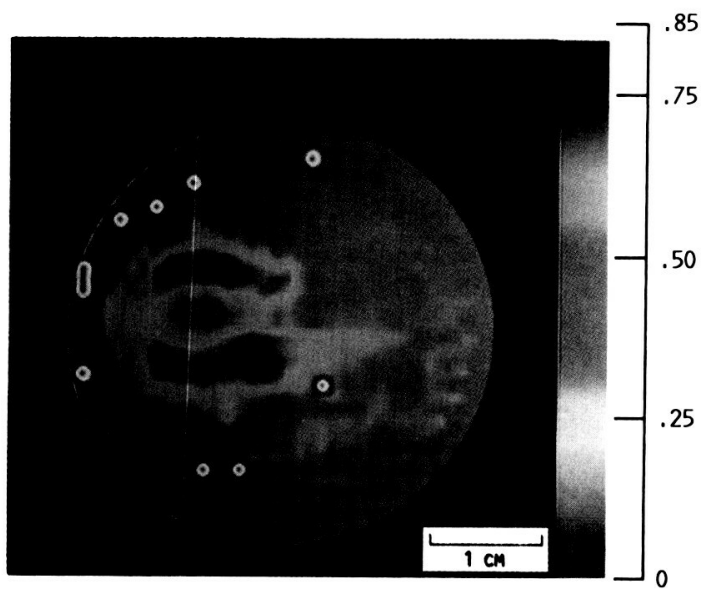

Figure 16.-Reflection coefficient map at $100 \mathrm{MHz}$ for $\mathrm{SiC}$ disk sintered at $2190{ }^{\circ} \mathrm{C}$

(CD-87-26965)

\section{Discussion}

The velocity and attenuation maps clearly indicate higher sensitivity to microstructural variations than the radiographic results. This is quite dramatic, particularly for the sample sintered at the lower temperature. The phase velocity maps revealed a more detailed image than the cross-correlation maps. This is a result of the averaging or smoothing effect implicit in the cross-correlation calculation where all frequency components of the waveforms are used to determine a single group-like velocity.

In the attenuation map (fig. 14) we have observed extremely low attenuation (at $100 \mathrm{MHz}$ ) at point $\mathrm{A}$ (most dense region) where there exist large grains. Therefore, grain boundary scattering can be ruled out as a dominant attenuation mechanism for frequencies up to $100 \mathrm{MHz}$ for $\mathrm{SiC}$ containing grain sizes ranging from 5 to $15 \mu \mathrm{m}$. This implies that each of the attenuation maps shown in this work is a map of attenuation due to scattering at the pore sites. Also at point A we have the highest velocity, therefore, increases in velocity correspond to higher densities.

Consider figures 5, 6, and 8 , at point $\mathrm{A}$ (with high velocity and high attenuation) we have a dense region with large pores, while at point B (with low velocity and low attenuation) we have a less dense region with smaller pores. The difference in porosity fraction between points $A$ and $B$ is 5 percent. The difference in the maximum pore size (excluding anomalously large pores) between points $A$ and $B$ is 500 percent. When comparing this to the variation in phase velocity of 4.5 percent and attenuation of 100 percent, between the two points, a general correlation is observed. The velocity is sensitive to the local porosity fraction and the attenuation is sensitive to the local pore size. That is, higher velocities correspond to denser regions and higher attenuation corresponds to regions with larger pores. So that for a "clean" ceramic having a very 
high density and small pore size we would expect to find very high velocity and low attenuation values. This is observed in the higher density sample at point $A$ in figures 12 to 14 . (It should be pointed out this is only a generalization and that pore clustering, pore size distribution, numerical number of pores, etc. may have to be considered.) These results are consistent with previous work (ref. 10). The actual correlation function is not known but may be obtained by determining the quantitative porosity size distribution function everywhere throughout the bulk of the sample. This is a formidable task requiring computer controlled, precision measurements of pore size.

\section{Conclusion}

Acoustic images of silicon carbide ceramic disk were obtained using a precision scanning contact pulse echo technique. Phase and cross-correlation velocity, and attenuation maps were used to form color images of microstructural variations. These acoustic images revealed microstructural variations not observable with $x$-ray radiography.

\section{References}

1. D.W. Richardson, Modern Ceramic Engineering: Properties, Processing and Use in Design, (Marcel Dekker, New York, 1982) Ch. 3.

2. S. Dutta, "Sinterability, Strength and Oxidation of Alpha Silicon Carbide Powders," J. Mater. Sci., 19, 1307 (1984).

3. S. Dutta, "Strength Optimization of a-SiC By Improved Processing," Structural Ceramics, NASA CP-2427, (NASA, Washington, D.C., 1986) pp. 89-98.

4. W.A. Sanders, and G.Y. Baaklini, "Correlation of Processing and Sintering Variables with the Strength and Radiography of Silicon Nitride," Ceram. Eng. Sci. Proc., 7, 839 (1986).

5. S.J. Klima, and G.Y. Baaklini, "Ultrasonic Characterization of Structural Ceramics," Analytical Ultrasonics in Materials Research and Testing, NASA CP-2383, (NASA, Washington, D.C., 1986) pp. 117-126.

6. G.Y. Baaklini, E.R. Generazio, and J.D. Kiser, "High Frequency Ultrasonic Characterization of Sintered SiC," presented at the 11th Annual Conference on Composition and Advanced Ceramic Materials, Jan. 18-23, 1987, Cocoa Beach, FL.

7. E.R. Generazio, "The Role of the Reflection Coefficient in Precision Measurement of Ultrasonic Attenuation," Mater. Eval. 43, 995 (1985)

8. D.R. Hull, H.E. Kautz, and A. Vary, "Measurement of Ultrasonic Velocity Using Phase-Slop and Cross-Correlation Methods," Mater. Eval, 43, 1455 (1985).

9. W. Sachse, and Y.H. Pao, "On the Determination of Phase and Group Velocities of Dispersive Waves in Solids,' J. Appl. Phys., 39, 4320 (1978).

10. A.G. Evans, B.R. Tittman, L. Ahlberg, B.J. Khuri-Yakub, and G.S. King, "Ultrasonic Attenuation in Ceramics," J. Appl. Phys. 49, 2669 (1978). 


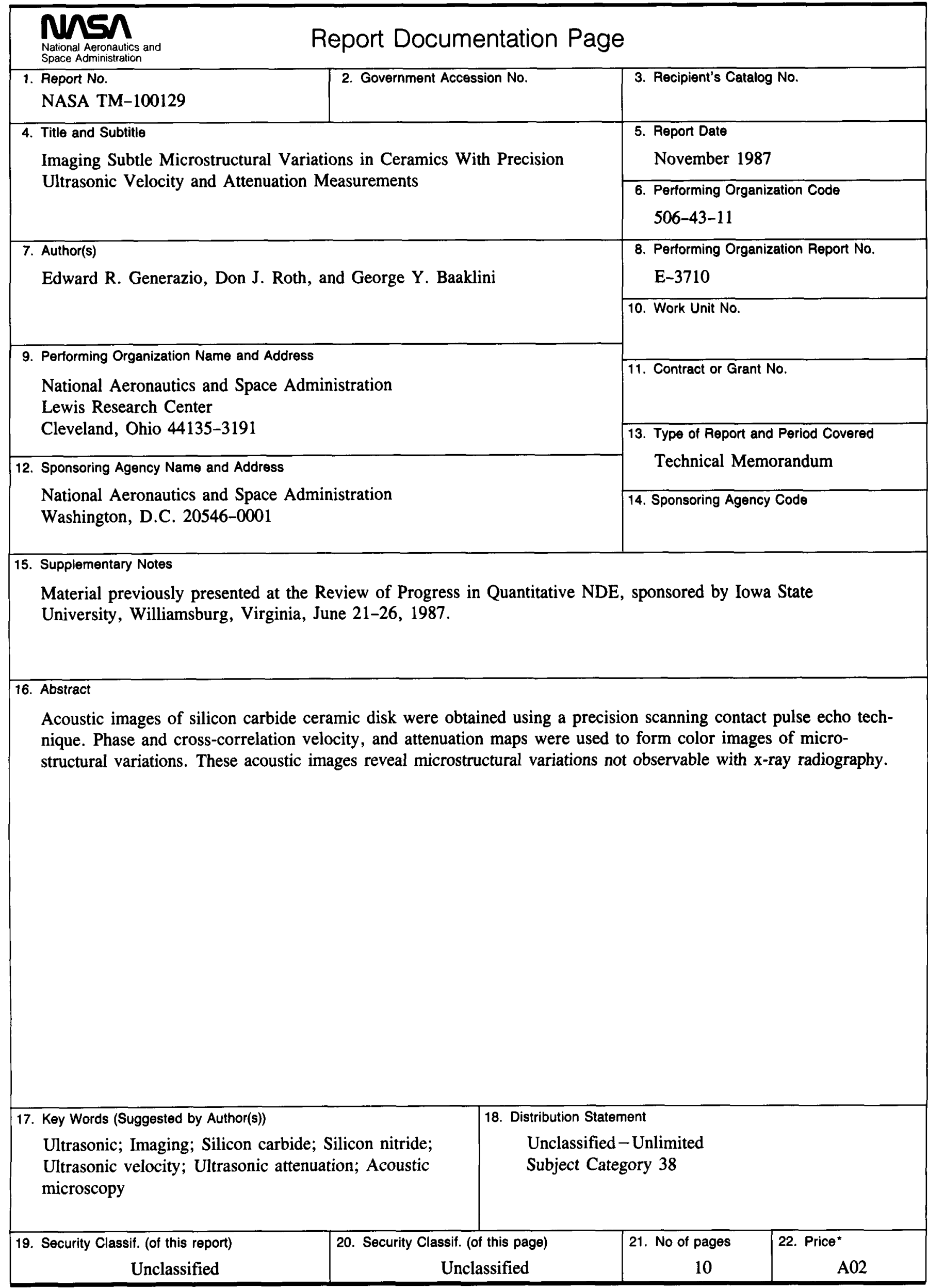

